\title{
Comparison of different Energy Minimization Techniques in Wireless Sensor Network
}

\author{
Sunita Gupta \\ CSE Deptt., JECRC University, Jaipur,
}

\author{
K.C.Roy, Ph.D \\ ECE Deptt., SBCET Jaipur, India.
}

\begin{abstract}
Wireless Sensor Networks (WSN) is a set of sensor nodes that collects the information from environment and sends to the base station (Header node or Central Node).Wireless range of applications related to national security, surveillance, home and office application[1],habitat monitoring[2,3],health application[4,5],environment forecasting[6] and military etc. One important class of WSNs is wireless Ad-Hoc sensor networks, characterized by an Ad-Hoc or random sensor deployment method, where the sensor location is not known a priori. This applies when individual sensor placement is infeasible, such as in battlefields or in disaster areas. Generally, more sensors are deployed than required (As compared with the optimal placement) to perform the proposed task; this compensates for the lack of exact positioning and improves fault tolerance. The characteristics of a sensor network include limited resources, large and dense networks, and a dynamic topology. An important issue in sensor networks is power scarcity, driven in part by battery size and weight limitations. Mechanisms that optimize sensor energy utilization have a great impact on prolonging the network lifetime. In this paper different energy minimization techniques have been compared that are used in wireless sensor network.
\end{abstract}

\section{Keywords}

Wireless sensor network, Energy efficient target coverage, Energy minimization, Lifetime of WSN, Network architecture, Cover set, Coverage, Connectivity.

\section{INTRODUCTION}

WSN are application specific and all design considerations are different for each application. The requirements of WSNs are very specific; especially when it comes to military application.WSN is limited in energy and has individual resources (such as CPU and memory). These tiny devices could be deployed in hundreds or even thousands in harsh and hostile environments. One of the advantages of wireless sensors networks (WSNs) is their ability to operate unattended in harsh environments in which contemporary human-in-the-loop monitoring schemes are risky, inefficient and sometimes infeasible. Therefore, sensors are expected to be deployed randomly in the area of interest by a relatively uncontrolled means, e.g. dropped by a helicopter, and to collectively form a network in an ad-hoc manner [7, 8]. Given the vast area to be covered, the short lifespan of the battery-operated sensors and the possibility of having damaged nodes during deployment, large population of sensors are expected in most WSNs applications. It requires scalable architectural and management strategies. In addition, sensors in such environments are energy constrained and their batteries cannot be recharged. The nodes lose their energy quickly and become dead. The frequent topology changes due to the die of sensors make the network quite unstable. In some application scenarios, replenishment of energy resources might be impossible, and therefore sensor node lifetime shows a very strong dependency on battery lifetime [9]. Therefore, designing energy-aware algorithms becomes an important factor for extending the lifetime of sensors.

\section{ENERGY RESOURCE IN WIRELESS SENSOR NODE}

In addition to a sensor, each node in a wireless sensor network is typically equipped with microcontroller, transceiver, sensors, memory, and power unit. The sensor node senses the environment, collects the relevant data, process the information collected, store it in a buffer and forward this information to other nodes or base station in a wireless manner. The processing unit is used to process incoming data and assemble them into packets to be transmitted using the wireless transceiver. An energy source (Power unit) supplies energy to the memory, sensing unit and transceiver.

Wireless sensor nodes must be supported by a power unit which is typically some form of storage (that is, a battery) but may be supported by power scavenging components (for example, solar cells). Energy from power scavenging techniques may only be stored in rechargeable (secondary) batteries and this can be a useful combination in wireless sensor environments where maintenance operations like battery changing are impractical. To conserve energy a power unit may additionally support power conservation techniques such as dynamic voltage scaling.

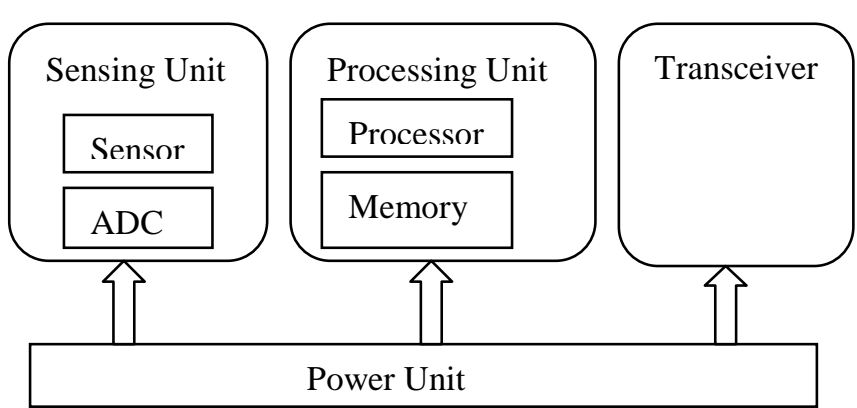

Fig 1: Components of Wireless Sensor Network 


\section{ENERGY WASTING REASONS IN WIRELESS SENSOR NETWORK}

The states of WSN are also important as sensor node consumes different amount of energy in different state. To increase the lifetime of WSN, changing the states according to the need is also important. In wireless sensor network each sensor nodes radio can be in one of the following four states

1) Transmit:-When the transmitter is transmitting.

2) Receive: - When the receiver is receiving.

3) Idle:-When the transceiver is neither transmitting nor receiving. It consumes less energy in idle state as compared to transmit and receive state

4) Sleep:- When radio is turned off. A sensor node does not consume any energy in sleep state.

In WSNs, sensors dissipate energy while sensing, processing, transmitting or receiving data to fulfill the mission required by the application. The sensing subsystem is devoted to data acquisition. It is obvious that minimizing data extracted from transducer will save energy of very constrained sensors. Experimental results confirm that communication subsystem is a greedy source of energy dissipation. A great amount of energy wasted in communication due to several reasons $[10,11]$ in states that are useless from the application point of view, such as:

Collision:-When a node receives more than one packet at the same time, these packets collide. All packets that cause the collision have to be discarded and the retransmission of these packets is required.

Overhearing:-When a sender transmits a packet, all nodes in its transmission range receive this packet even if they are not the intended destination. Thus, energy is wasted when a node receives packets that are destined to other nodes.

Control packet overhead: - A minimal number of control packets should be used to enable data transmissions.

Idle listening:-It is one of the major sources of energy dissipation. It happens when a node is listening to an idle channel in order to receive possible traffic.

Overmitting:- It happens when a sender sends packet to a node but the receiver node is not ready and the packet must be sent again.

Interference:-Each node located between transmission range and interference range receives a packet but cannot decode it.

\section{ENERGY SAVING TECHNIQUES IN WSN}

As battery is the only source of energy in WSN and it cannot be recharged. So powers saving techniques are used to conserve the energy in WSN. For a wireless sensor node, power saving techniques can generally be classified in the following categories.

1) Schedule the wireless nodes to alternate between active and sleep mode.

2)Power control by adjusting the transmission range of the wireless nodes.

3)Energy efficient routing and data gathering.
Reduce the amount to data transmitted and avoid useless activity.

4)These power saving techniques can be used in clustering, routing or scheduling of nodes techniques to increase the lifetime of WSN

\section{CLUSTERING IN WSN}

Grouping sensor nodes into clusters has been widely used by the research community in order to achieve the network scalability objective, energy efficiency, effective data communication and robustness for the network by allowing spatial reuse of the bandwidth and simpler routing decisions that results in decreased energy dissipation of the whole system by minimizing the number of nodes that takes part in long distance communication[12] .Every cluster would have a leader, often referred to as the cluster-head $(\mathrm{CH})$ as shown in figure 2. Cluster heads collect, aggregate and forward the data. Communications are either intra cluster, that is, between nodes and cluster head inside a cluster or inter cluster which can occur between one node in a cluster and a different node in another cluster. In later case, cluster heads play a prominent role in successful communication. In most clustering approaches, first a set of cluster heads are selected among the nodes in the network. After that rest nodes are clustered around these CHs. Selecting cluster heads is always a hard problem. Generally clustering approaches use random scheme to select cluster heads. A comparison various clustering algorithms used in wireless sensor networks is given in [13]. Clustering schemes is classified into four categories - heuristic, hierarchical, weighted, and grid scheme [14]. Clustering algorithms play a vital role in achieving the targeted design goals for a given implementation.

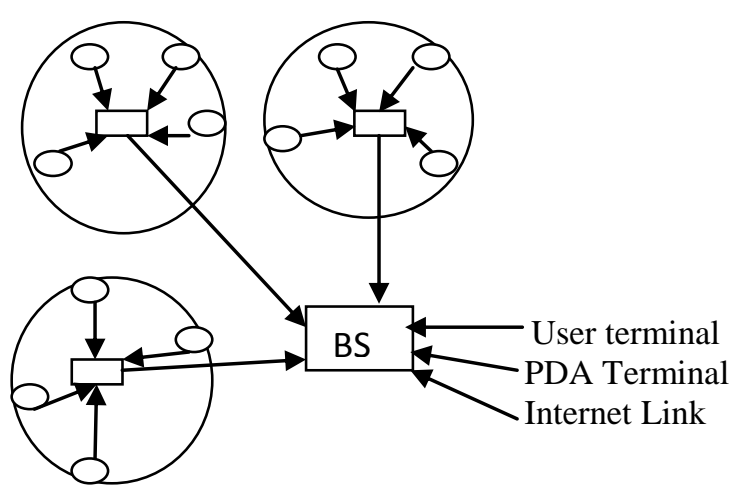

Sensor

Cluster Head

Fig.2: General clustered sensor network architecture

There are several key attributes that designers must carefully consider while clustering, which are of particular importance in wireless sensor networks.

- Cost of Clustering: Although clustering plays a vital role in organizing sensor network topology, there are often many resources such as communication and processing tasks needed in the creation and maintenance of the clustering topology. 
- Selection of Cluster heads and Clusters: For designing WSN for a particular application, designers must carefully examine the formation of clusters in the network. Depending on the application, certain requirements for the number of nodes in a cluster or its physical size may play an important role in its operation. This prerequisite may have an impact on how cluster heads are selected in this application.

- Real-Time Operation: Useful lifetime of data is also a fundamental criterion in designing Wireless Sensor Networks. In applications such as habitat monitoring [2, 3], simply receiving data is sufficient for analysis. Means delay is not an important issue. In military tracking [15], the issue of real-time data acquisition becomes much more vital. So, important attention must be paid to the delay created by the clustering scheme itself. In addition, the time required for cluster recovery mechanisms must also be taken into account.

- Synchronization: One of the primary limitations in Wireless Sensor Networks is the limited energy capacity of nodes. Slotted transmission schemes (such as TDMA), allow nodes to regularly schedule sleep intervals to minimize energy used. Such schemes require synchronization mechanisms to setup and maintain the transmission schedule. When considering a clustering scheme, synchronization and scheduling will have a considerable effect on network lifetime and the overall network performance.

- Data Aggregation: Data aggregation allows the differentiation between sensed data and useful data. As the power required for processing tasks is substantially less than communication tasks, the amount of data transferred in network should be minimized. Many clustering schemes provide data aggregation capabilities, and as such, the requirement for data aggregation should be carefully considered when selecting a clustering approach.

- Repair Mechanisms: Nodes in WSN are often prone to node mobility, node death and interference. All of these situations can result in link failure. When looking at clustering schemes, it is important to look at the mechanisms in place for link recovery and reliable data communication.

- Quality of Service (QoS): There are many QoS requirements in WSN. Many of these requirements are application dependant (such as acceptable delay and packet loss tolerance), and as such, it is important to look at these metrics when choosing a clustering scheme. Implementations can vary widely in terms of these metrics, The information collected by sensor nodes can be relayed back to data sink or controllers. Therefore connectivity is as critical as sensing coverage. Coverage means the area that has to be monitored by the sensor nodes. Life time of WSN means the amount of time for which the given sensor network remain active and provide sufficient information of the coverage area. It is understood that every target in the network should be covered by more than one node so that it may remain connected even if one sensor fails. Higher order of communication is also required for appropriate communications. So there is requirement of Q-Coverage and $\mathrm{P}$-connectivity. Q-coverage means every point in the plane is covered by at least q-different sensors [27]. Pconnectivity means there are at least $p$ disjoint paths between any two sensors [27]. Maximizing the network and as a result, the design process should consider these aspects.

Cluster formulation is a 2 step process. First cluster head is elected and then nodes are assigned to cluster head $[16,17]$. So cluster head energy consumption should be minimized and thus the network lifetime is maximized. There are many energy efficient data collecting clustering protocols. LEACH (Low energy adaptive clustering hierarchy) is a clustering protocol where cluster head is selected randomly, to balance the energy of the network [18]. Cluster head accept the data from all sensors of the same cluster aggregate them and then finally send to the base station. All cluster heads are in direct communication with BS.As cluster heads are selected randomly, this protocol has a probability of having a low energy node as a cluster head. At this condition $\mathrm{CH}$ is not able to communicate with BS, for a long time. Thus network lifetime decrease and it will affect network performance. To solve this problem SEP (Stable election protocol) was developed [19].In SEP protocol, sensors having high energy have more probability of becoming $\mathrm{CH}$. It greatly increases the stable reason as compared to LEACH. In WSN energy efficiency and low latency are two main issues. To achieve these HEARP (Hierarchical energy aware routing protocol )was developed[20].It was batter then LEACH in terms of energy consumption and latency. HEARP saves energy and increase the stability period of WSN.

Then PEGASIS was developed to increase the stability period of WSN and lifetime of WSN [21]. After that chain based clustering routing protocol called WEP (Weighted election protocol) was developed. Here nodes are divided into 2 types. Advanced nodes and normal nodes. Advanced nodes have higher energy than normal nodes. Advanced nodes become $\mathrm{CH}$ more often as compared to normal nodes. It combines the clustering strategy with routing algorithm to satisfy both energy and stable period constraints in the heterogeneous environment [22].

Many other algorithms have also been developed for this like ANDA [23], LID [24, 25], LEAD [26] etc.

\section{ENERGY EFFICIENT SCHEDULING}

Coverage and Connectivity are basic requirements in a wireless sensor network. The objective of such a network is to detect events of interests or collect data and then report the obtained information to a fusion centre. Connectivity means the sensor network remain connected so

lifetime and to provide reliable energy efficient monitoring depends on selecting minimum number of sensors in active mode to cover all the targets. So these power saving techniques can be regarded as Set Covering Problem (SCP). The sensors from the active set are in the active state (e.g. transmit, receive or idle) and all other sensors are in the sleep state. In the active mode, a sensor can observe the environment and communicate with other sensors (or the base station). In the sleep mode, a sensor cannot monitor or communicate. However, the node can change to the active mode, any time it receives the appropriate signal (either from another sensor or the base station). Obviously, in the sleep mode a sensor consumes much less energy than in active mode. Finally, in the off mode, the nodes are completely inactive. This happens either when the battery of 
a sensor is exhausted or when the sensor is turned off completely. Sensors have a limited battery life. In order to increase the lifetime, the nodes must be divided into a number of subsets, called cover sets. Sensors belonging to the currently scheduled cover set are in active mode, while others are in sleep mode. Each cover set is capable of covering all monitored targets. If the cover sets are nodedisjoint sets, then each sensor will be allowed to participate only in one cover set, and thus each set will have a maximum lifetime of $\mathrm{k}$. By periodically switching between sets of coverage sensors, the target coverage time can be extended to $\mathrm{s} \cdot \mathrm{k}$, where $\mathrm{s}$ is the number of available sets. There are many techniques of solving the problem of active node selection in WSNs [28, 29], and most of them having aim of achieving high levels of energy efficiency. To select optimal cover set any algorithms may be used like efficient cover set selection (ECSS)[30], which is based on improved NSGA-II (elitist non dominated sorting genetic algorithm) and it gives optimal number of set covers. Basic Ant Colony Algorithm [31] is used to solve the SCP. Here the minimum number of sets of sensors is selected where at least one sensor is BS connected to prolong the network lifetime. In the modified Ant Colony Algorithm [32] set covering problem is solved where node selection procedure is based on the energy of each node in a set, provide energy efficient sensor network.

Greedy-MSC Heuristic for 1-Coverage as well as for QCoverage is used and defined in [33].It generates nondisjoint cover sets and efficiently increases the lifetime. An Adaptive heuristic also known as $\mathrm{B}\{\mathrm{GOP}\}$ was proposed[34].It is centralized algorithm that generates nodedisjoint sensor sets, each capable of independently monitoring all targets. In this novel centralized algorithm each sensor set is capable of monitoring independently all registered targets. The algorithm provides efficient solutions for both point and area coverage problems. A Heuristic with high energy and small lifetime (HESL) [35], is proposed to increase the lifetime of WSN by considering Q-Coverage requirement. This algorithm use three steps.

1)Priority assignment - Priorities are assigned to each sensor based on available battery life. Thus a Q-Cover is generated by selecting sensors with highest residual battery life till all the targets are covered according to Q-Coverage vector. By this the obtained Q-Cover which is not minimal. To find out minimal Q-cover one sensor is removed at a time and check whether it is a Q-Cover or not.

2)Life time assignment $-A$ small constant of lifetime is assigned to the set cover $\mathrm{C}$, thus generated. So one sensor may participate in more than one cover set as a sensor doesn't consume all of its energy in a single cover set. This constant lifetime is decided as minimum between small lifetime granularity constant (l) and maximum lifetime available of sensors in a set cover $c_{p}$.

3)Priority decrement - To avoid the repeated generation of same Q-Cover always the priority of each sensor is reduced by one, once it is used in a Q-Cover. So after a sensor is used in a set cover, its priority reduces and thus chances are given to other nodes also to participate in set cover by increasing their priority.

\section{ROUTING IN WSN}

Routing in a communication network is the process of forwarding a message from a source host to a destination host via intermediate nodes. In wired networks, routing is commonly a task performed by routers. In ideal wireless ad hoc networks, in contrast, every network node may act as a router, as a relay node forwarding a message on its way from its source node to its destination node. This process is particularly important in ad hoc networks, as network nodes are assumed to have restricted power resources and therefore try to transmit messages at low transmission power, leading to the effect that the destination of a message can typically not be reached directly from the source. In wired networks, routing almost always takes place in relatively stable conditions; at least the main neighborhood topology remains identical over weeks, months, or even years. The primary focus of routing in wired networks is on high-performance forwarding of messages; reaction latency in the face of network topology changes, caused by failing hosts or connections, is generally of secondary importance. Considering the stability of wired networks, prompt reaction to topology changes or rapid propagation of according information is often not required; as such events are relatively rare. A considerable number of routing protocols specifically devised for operation in ad hoc networks have consequently been invented. Routing protocols are usually classified into two groups: proactive and reactive routing protocols. Proactive routing protocols resemble protocols for wired networks in that they collect routing information ahead of time. A request for a message to be routed can be serviced without any further preparative actions. As every node keeps a table specifying how to forward a message, information on topology changes is propagated whenever they occur. Similar to routing protocols in wired networks, proactive routing protocols are efficient only if links are stable and node mobility is low compared to the rate of communication traffic. Already if node mobility reaches a reasonable degree, the routing overhead incurred by table update messages can become unacceptably high [36]. Another question is whether lightweight ad hoc network nodes with scarce resources can be expected to maintain routing tables potentially for all possible destinations in the network. Reactive routing protocols, on the other hand, try to delay any preparatory actions as long as possible. Routing occurs on demand, only. In principle, a node wishing to send a message has to flood the network in order to find the destination. Although there are many tricks to restrict flooding or to cache information overheard by nodes, flooding can consume a considerable portion of the network bandwidth. Attempting to combine the advantages of both concepts, proposals have also been made to incorporate both approaches in hybrid protocols, adapting to current network conditions. Proactive protocols, such as DSDV [37], TBRPF [38], and OLSR [39], distribute routing information ahead of time in order to be able to react immediately whenever a message needs to be forwarded. On the other hand, reactive protocols, such as AODV [40], DSR [41]do not try to anticipate communication initiate route discovery as late as possible, as a reaction to a message requested to be routed. Some hybrid protocols, such as [43, 44], have been proposed. Further details of routing algorithms in mobile ad hoc networks are given in [45]. Most of these protocols have been described and studied from a system perspective; performance and efficiency assessment was commonly carried out by means of simulation. Greedy routing and face routing can be combined, resulting in the GOAFR [46] algorithm, which preserves the worst-case guarantees of its face routing components. GOAFR particularly outperforms other routing algorithms in a critical node density range, where the network is just about to become connected and 
which forms a challenge to any routing algorithm, also nongeographic routing algorithms.

\section{PROPOSED APPROACH FOR ENHANCING ENERGY IN WSN}

Lifetime of battery is limited. It cannot be used them for loner time. Other approaches can also be used like energy efficient clustering, routing and scheduling for WSN Energy efficient scheduling for sensor nodes is used in such a way that all nodes are not activated at the same time and thus energy consumption is minimized. Means some nodes are in active state while some are in sleep state. The lifetime of WSN depends upon battery consumption. Lifetime means the amount of time for which the given sensor network remain active and provide sufficient information of the coverage area. A network is connected if every node in the network is part of the mainland and not connected if at least one island exists. The mainland is part of the network which contains the sink together with the sensor nodes those are connected to the sink, either directly or via other nodes. Sensor nodes in a mainland can send their messages to the sink, by definition. An island in the network contains one or more nodes which are not connected to the sink; hence, they can send messages to each other, but cannot send any message to the sink. This is the main drawback of wireless Sensor Network. To remove the problem of battery in Wireless sensor network a new mechanism can be used which may activates the battery (sensor) and thus their battery is not exhausted. If sensor node can be charged by using some mechanism then they can be used for longer time and there is no need to be care about node scheduling for saving battery power. This mechanism can solve the problem of energy in wireless sensor node.

Energy source can be used in the form of sun or wind. By using sun energy in the day time, all batteries of sensor nodes in the network are activated. In day time sensors are using energy directly from the sun and in the night they can use stored sun energy in their batteries. But in case of dense forest sun availability may not be proper or even impossible. Thus such energy supplies are not reliable. So at those places this mechanism will not work

\section{ACKNOWLEDGEMENTS}

We would like to thank to Mr. Sakar Gupta for fruitful discussions and for providing the initial insights regarding Wireless Sensor Network and different energy issues of WSN.

\section{REFERENCES}

[1] Mani B. Srivastava, Richard R. Muntz, and Miodrag Potkonjak. Smart kindergarten: sensorbased wireless networks for smart developmental problem-solving environments. In Mobile Computing and Networking, pages $132.138,2001$

[2] A. Cerpa, J. Elson, D. Estrin, L. Girod, M. Hamilton, and J. Zhao. Habitat monitoring:Application driver for wireless communications technology. In Proceedings of the 2001ACM SIGCOMM Workshop on Data Communications in Latin America and the Caribbean, April 2001., 2001.

[3] Alan Mainwaring, Joseph Polastre, Robert Szewczyk,David Culler, and John Anderson. Wireless sensor networks for habitat monitoring. In ACM
International Workshop on Wireless Sensor Networks and Applications (WSNA'02), Atlanta, GA, September 2002.

[4] I. F. Akyildiz, W. Su, Y. Sankarasubramaniam and E.Cayirci, "A Survey on Sensor Networks", IEEE Communications Magazine, (Aug. 2002), pp 102-11.

[5] Loren Schwiebert, Sandeep K. S. Gupta, and Jennifer Weinmann. Research challenges in wireless networks of biomedical sensors. In Mobile Computing and Networking, pages151. 165, 2001.

[6] Edoardo Biagioni and Kent Bridges. The application of remote sensor technology to assist the recovery of rare and endangered species. In Special issue on Distributed Sensor Networks for the International Journal of High Performance Computing Applications, Vol. 16, N. 3 , August 2002.

[7] K. Sohrabi et al., Protocols for self-organization of a wireless sensor network, IEEE Personal Communications 7 (5) (2000) 16-27.

[8] R. Min, et al., Low power wireless sensor networks, in: Proceedings of International Conference on VLSI Design, Bangalore, India, January 2001.

[9] V. Kawadia, P.R. Kumar, Power control and clustering in Ad Hoc networks, in: Proceedings of IEEE INFOCOM, San Francisco, CA, March 2003.

[10] Y. Wei, J. Heidemann et al., "An energy-efficient MAC protocol for wireless sensor networks," in Proceedings of the 21st Annual Joint Conference of the IEEE Computer and Communications Societies (INFOCOM '02), pp. 1567-1576, New York, NY, USA, June 2002.

[11] H. Y. Shwe, X.-H. Jiang,, and S. Horiguchi, "Energy saving in wireless sensor networks," Journal of Communication and Computer, vol. 6, no. 5, pp. 20-27, 2009

[12] W. Liu and J. Yu, "Energy efficient clustering and routing scheme for wireless sensor networks," inProceedingsof the IEEE International Conference on Intelligent Computing and Intelligent Systems (ICIS '09), vol. 3, pp. 612-616, 2009.

[13] L. M. Arboleda C and N. Nasser, "Comparison of clustering algorithms and protocols for wireless sensor networks," in Proceedings of the Canadian Conference on Electrical and Computer Engineering (CCECE '06), pp. 1787-1792, May 2006

[14] D. J. Dechene, A. El Jardali, M. Luccini, and A. Sauer, "A survey of clustering algorithms for wireless sensor networks," Project Report, 2006.

[15] C.Y.Chong, S.Mori, and K.C.Chang, "Distributed multitarget multisensory tracking," in Multitarget Multisensor Tracking:Advanced Applications, 1990.

[16] Kwon, T., Gerla, M.: Clustering with Power Control, Proc.MILCOM’99,November (1999).

[17] Heinzelman, W.B., Chandrakasan, A., Balakrishnan, H.: Energy-Efficient Communication Protocols for Wireless Microsensor Networks.In Proceedings of Hawaiian InternationalConference on Systems Science, January (2000) 
[18] W. Heinzelman, A. Chandrakasan, H. Balakrishnan, "Energy-Efficient Communication Protocol for Wireless Microsensor Networks", HICSS 2000.

[19] Georgios Smaragdakis, Ibrahim Matta \& Azer Bestavros, (2004) "SEP: A Stable Election Protocol for Clustered heterogeneous wireless Sensor Networks", Second International Workshop on Sensor and Actuator Network Protocols and Applications (SANPA-04).

[20] Shamsad Parvin, (2007) "Hierarchical Energy Aware Routing Protocol (HEARP) for Wireless Sensor Networks", MSc Thesis, University of Rajshahi, Bangladesh.

[21] S. Lindsey, C. S. Raghavendra, PEGASIS: "Power Efficient Gathering in Sensor Information Systems", IEEE Aerospace Conference (Mar. 2002).

[22] Md. Golam Rashed1, M. Hasnat Kabir2, Shaikh Enayet Ullah3," WEP: AN ENERGY EFFICIENT PROTOCOL FOR CLUSTER BASED HETEROGENEOUS WIRELESS SENSOR NETWORK", International Journal of Distributed and Parallel Systems (IJDPS) Vol.2, No.2, March 2011.

[23] Chiasserini, C.F.,Chlamtac, I.,Monti,P.,Nucci,A.: Energy Efficient Design of wireless ad-hoc network.LNCS 2006, vol. 2345, pp. 376-386.

[24] Ephremides, A., Wieselthier, J.E., Baker, D.J.: Proc.IEEE, VOL.75, NO.1, January(1987).

[25] Baker, D.J., Ephremides, A.: The architectural organization of a mobile radio network via a distributed algorithm.In: IEEE Transactions on Communications, pp. 1694-1701, November (1981)

[26] Subhankar Mishra1, Sudhansu Mohan Satpathy1 and Abhipsa Mishra," ENERGY EFFICIENCY IN AD HOC NETWORKS", International Journal of Ad hoc, Sensor \& Ubiquitous Computing (IJASUC) Vol.2, No.1, March 2011

[27] X. Bai, S. Kumar, D. Xuan, Z. Yun and T. H. Lai "Deploying Wireless Sensors to Achieve Both Coverage and Connectivity". In Proc. of ACM MobiHoc, 2006.

[28] M. Perillo, W. Heinzelman, "Optimal sensor management under energy and reliability constraints", in: Proc. of the IEEE Wireless Communications and Networking Conference, New Orleans, Louisiana, USA, March 2003.

[29] S. Meguerdichian, M. Potkonjak, "Low power 0/1 coverage and scheduling techniques in sensor networks", UCLA Technical Reports 030001, January 2003.

[30] JIA Jie1 CHEN Jian1, CHANG Gui-Ran2 ,WEN Ying-You1," Efficient Cover Set Selection in Wireless Sensor Networks", Acta Automatica sinila Vol.34,No.9 September,2008.

[31] Ricardo M Silva, A De, Geber L Ramalho. (2001) "Ant system for the set covering problem".Systems, Man, and Cybernetics, IEEE International Conference, Vol 5 pp. 3129-3133.

[32] Salma Begum, Nazma Tara, Sharmin Sultana , “ Energy-Efficient Target Coverage in Wireless Sensor Networks Based on Modified Ant Colony Algorithm",
International Journal of Ad hoc, Sensor \& Ubiquitous Computing (IJASUC) Vol.1, No.4, December 2010,

[33] Mihaela Cardei, My T. Thai ,Yingshu Li ,Weili Wu, "Energy Efficient TargetCoverage Problems in Wireless Sensors Networks", "IEEE INFOCOM 2005

[34] Dimitrios Zorbas, Dimitris Glynos, Panayiotis Kotzanikolaou Christos Douligeris, "An Adaptive Algorithm for Coverage Problem in Wireless Sensor Networks", "IEEE INFOCOM'05 March".

[35] Manju Chaudhary and Arun K Pujari, "Q-coverage Problem in Wireless Sensor Networks",Published in: - Proceeding ICDCN '09 Proceedings of the 10th International Conference on Distributed Computing and Networking Pages 325 - 330

[36] J. Broch, D. Maltz, D. Johnson, Y.-C. Hu, and J. Jetcheva, "A performance comparison of multi-hop wireless ad hoc network routing protocols," in Proc. ACM MobiCom, 1998, pp. 85-97 [Online]. Available:citeseer.nj.nec.com/broch98performance.htm 1

[37] C. Perkins and P. Bhagwat, "Highly dynamic Destination-Sequenced Distance-Vector Routing (DSDV) for mobile computers," in Proc.ACM SIGCOMM'94, 1994, pp. 234-244.

[38] R. Ogier, F. Templin, and M. Lewis, "Topology Dissemination Basedon Reverse-Path Forwarding (TBRPF)," IETF RFC 3684, Feb. 2004

[39] T. Clausen and P. Jacquet, "Optimized Link State Routing Protocol (OLSR)," IETF RFC 3626, Oct. 2003.

[40] C. Perkins and E. Royer, "Ad-hoc on-demand distance vector routing,"in Proc. 2nd IEEE Workshop on Mobile Computing Systems and Applications,1999, pp. 90100 .

[41] D. Johnson and D. Maltz, "Dynamic source routing in ad hoc wireless networks," in Mobile Computing, Imielinski and Korth, Eds. Boston,MA: Kluwer Academic, 1996, vol. 353 [Online]. Available: citeseer.nj.nec.com/johnson96dynamic.html

[42] V. Park and M. Corson, "A highly adaptive distributed routing algorithm for mobile wireless networks," in Proc. 16th Annu. Joint Conf. IEEE Computer and Communications Societies (INFOCOM),1997, pp. 1405-1413 [Online]. Available: citeseer.ist.psu.edu/article/park97highly.html

[43] N. Nikaein, C. Bonnet, and N. Nikaein, "HARPHybrid Ad Hoc Routing protocol," in Int. Symp Telecommunications (IST 2001),Tehran, Iran, Sep. 2001.

[44] V. Ramasubramanian, Z. Haas, and E. Sirer, "SHARP: A hybrid adaptive routing protocol for mobile ad hoc networks," in Proc. ACM MobiHoc,2003.

[45] E. Royer and C. Toh, "A review of current routing protocols for ad hoc mobile wireless networks," IEEE Personal Commun., vol. 6, no. 2, pp. 46-55, Apr. 1999.

[46] Fabian Kuhn, Member, IEEE, Roger Wattenhofer, and Aaron Zollinger, Member, IEEE, "An Algorithmic Approach to Geographic Routing in Ad Hoc and 
International Journal of Computer Applications (0975 - 8887) Volume 75-No.18, August 2013 